Research Article

\title{
State biomarkers for Machado Joseph disease: Validation, feasibility and responsiveness to change
}

Gabriel Vasata Furtado ${ }^{1,2,3^{*}}$, Camila Maria de Oliveira ${ }^{3,4,5^{*}}$, Gabriela Bolzan ${ }^{1,3,4^{*}}$ iD, Jonas Alex Morales Saute $^{3,4,5,7}$, Maria Luiza Saraiva-Pereira ${ }^{1,2,3,6}$ iD and Laura Bannach Jardim ${ }^{1,3,4,5,7}$ iD

${ }^{1}$ Programa de Pós-Graduação em Genética e Biologia Molecular, Universidade Federal do Rio Grande do Sul (UFRGS), Porto Alegre, RS, Brazil.

${ }^{2}$ Laboratório de Identificação Genética, Hospital de Clínicas (HCPA), Porto Alegre, RS, Brazil.

${ }^{3}$ Serviço de Genética Médica, Hospital de Clínicas de Porto Alegre (HCPA), Porto Alegre, RS, Brazil.

${ }^{4}$ Faculdade de Medicina, Universidade Federal do Rio Grande do Sul (UFRGS), Porto Alegre, RS, Brazil.

${ }^{5}$ Programa de Pós-Graduação em Medicina: Ciências Médicas, Universidade Federal do Rio Grande do Sul (UFRGS), Porto Alegre, RS, Brazil.

${ }^{6}$ Departamento de Bioquímica, Universidade Federal do Rio Grande do Sul (UFRGS), Porto Alegre, RS, Brazil.

${ }^{7}$ Departamento de Medicina Interna, Universidade Federal do Rio Grande do Sul (UFRGS), Porto Alegre, RS, Brazil.

\begin{abstract}
Machado-Joseph disease (SCA3/MJD) is the most common spinocerebellar ataxia worldwide, and particularly so in Southern Brazil. Due to an expanded polyglutamine at ataxin-3, SCA3/MJD presents a relentless course with no current disease modifying treatment. Clinical scales used to measure SCA3/MJD progression present moderate effect sizes, a major drawback for their use as main outcomes in clinical trials, given the rarity and slow progression of the disease. This limitation might be overcome by finding good surrogate markers. We present here a review of studies on peripheral and neurophysiological markers in SCA3/MJD that can be candidates for state biomarkers. Data on markers already studied were summarized, giving emphasis on validation against clinical scale, and responsiveness to change. While some biological fluid compounds and neurophysiological parameters showed poor responsiveness, others seemed to be good candidates. Some potential candidates that are waiting for responsiveness studies were serum levels of neuron specific enolase, vestibulo-ocular reflex and video-oculography. Candidates evaluated by RNA and microRNA expression levels need further studies to improve their measurements. Data on peripheral levels of Beclin-1 and DNAJB1 are promising but still incipient. We conclude that several potential candidates should follow onto validating studies for surrogate state biomarkers of SCA3/MJD.
\end{abstract}

Keywords: Biomarkers, neurophysiology, Machado-Joseph disease, spinocerebellar ataxia type 3.

Received: April 24, 2018; Accepted: November 4, 2018.

\section{Introduction}

Machado-Joseph disease, also known as spinocerebellar ataxia type 3 (SCA3/MJD), is an autosomal dominant spinocerebellar ataxia caused by an expanded $\mathrm{CAG}$ repeat (longer than 51 triplets) at $A T X N 3$ gene, giving rise to an expanded polyglutamine (polyQ) at ataxin-3 protein (Saute and Jardim, 2015). With a mean age at onset of 34-40 yo (Dürr et al., 1996; Schöls et al., 1997; Tang et al.,

Send correspondence to Laura Bannach Jardim. Medical Genetics Service Hospital de Clínicas de Porto Alegre, Rua Ramiro Barcelos 2350, 90035-003 Porto Alegre, RS, Brazil. E-mail: ljardim@ @cpa.edu.br.

${ }^{*}$ These authors contributed equally to this work.
2000; Globas et al., 2008; de Castilhos et al., 2014; du Montcel et al., 2014; Zhou et al., 2014), SCA3/MJD involves predominantly the cerebellar, pyramidal, extrapyramidal, motor neuron, and oculomotor systems. Gait ataxia is commonly the first symptom, followed by diplopia, dysarthria, spasticity, dystonic movements, sensory losses and other findings, in different combinations (Jardim et al., 2001; Saute and Jardim, 2015). SCA3/MJD is very heterogeneous and never exclusively ataxic. Currently there is no disease modifying treatment and SCA3/MJD presents a relentless progression, with an average survival of 21.18 years after onset of symptoms (Kieling et al., 2007). However, several lines of pre-clinical research gave rise to good candidate treatments targeting different cellular and molec- 
ular pathways, a scenario in which robust designs of clinical trials will be paramount for the success of the therapeutic endeavor (Li et al., 2015; Duarte-Silva et al., 2018; Matos et al., 2018). Considering the very slow progression of SCA3/MJD on clinical scales and the rarity of the disease, state biomarkers might be important surrogate endpoints for these future clinical studies.

Biomarkers are substances, structures, or processes that can be measured in the body or its products and influence or predict the incidence or outcome of disease, of treatments, or of environmental exposures" (WHO International Programme on Chemical Safety, 2001). Trait biomarkers are present prior to start of the disease process, while state biomarkers are due to disease process or due to a therapy response, and mirror disease progression. State biomarkers should be correlated to clinically meaningful endpoints. If state biomarkers show advantages when comparing to clinical endpoints, they can replace them in clinical trials (Aronson, 2005). This is the case of a biomarker whose changes can be measured easily and in a more sensitive way than clinical endpoints. Such surrogate markers are especially important for phase II, randomized clinical trials (phase II RCT) addressed to raise preliminary evidence of efficacy for a given drug, especially in the context of rare diseases.

Efficacy of a given treatment is most fully demonstrated when outcomes of treated versus control groups vary according to a minimal clinically important difference (MCID); and MCID were never clearly determined to SCA3/MJD. The closest to that was obtained by the Scale of Assessment and Rating of Ataxia (SARA), a validated semi-quantitative scale that progresses between 0.65 and 1.56/40 points per year (Schmitz-Hübsch et al., 2006, 2010; Chan et al., 2011; Ashizawa et al., 2013; Jacobi et al., 2015), and where 1.5 points were noted by patients according to the patients global impression of improvement (PGI-I). Nevertheless, disease progression is slow as measured by SARA and by all other clinical scales in use - the International Cooperative Ataxia Rating Scale (ICARS) (Trouillas et al., 1997), Neurological Examination Score for Spinocerebellar Ataxias (NESSCA) (Kieling et al., 2008), Composite-Cerebellar-Functional-Score (CCFS) (du Montcel et al., 2008), and the Inventory of Non-Ataxia Symptoms (INAS) (Schmitz-Hübsch et al., 2008). Clinical trials should be tailored to face this issue.

A drawback shared by all clinical scales is their large variability, which can reduces their effect sizes (ES), either by the Cohens effect size (CES), or the standardized response mean (SRM) (Streiner and Norman, 2008; Saute et $a l ., 2012)$. The average SRM obtained for SARA scale was 0.5 (Schmitz-Hübsch et al., 2010). Considering SARA SRM with a progression of 1 point per year, between 175 and 328 subjects would be needed in each arm to show a $50 \%$ reduction in the disease progression rate in a future trial (Schmitz-Hübsch et al., 2010; Chan et al., 2011; Saute et al., 2015). For a rare disease, these numbers are generally unfeasible. This might be overcome by the discovery of a good surrogate, or a set of surrogate markers, with ES larger than those presented by current clinical scales.

Since biomarkers are much needed, we aimed to review the state of art of potential surrogate markers of disease state in SCA3/MJD, focusing on neurophysiology markers and biological fluid compounds. Candidates for state biomarkers were included, provided that some preliminary evidence in humans was already published. Validation against a meaningful clinical endpoint, feasibility, rate of change in time (progression rate), and responsiveness to change were the parameters in focus.

\section{Materials and Methods}

\section{Search methods}

We performed a search in MEDLINE up to November, 2017. The search terms were (Machado-Joseph disease OR spinocerebellar ataxia) AND (Biomarker* OR Biologic* Marker* OR Laboratory Marker* OR Serum Marker* OR Surrogate Endpoint* OR Biochemical Marker* OR Immune Marker* OR immunologic* marker* OR miRNA) OR (Biomarker* OR Electroencephalography* OR Evoked potentials* OR Transcranial Magnetic Stimulation* OR Quantitative Motor Features* OR Vestibular* OR Video-Oculography* OR Nerve Conduction Studies* OR Electromyography*).

In addition, a manual search for references known by authors that were not covered by the above search strategy was also performed, and such studies were included.

\section{Criteria for including studies}

We included studies describing biological fluid compounds and neurophysiological measures that could be candidate for state biomarkers. Case-control and prospective studies and clinical trials were also included, provided that quantitative information on their candidate markers were given.

Original studies on cellular or animal models, as well as studies in humans lacking quantitative data, or when specific SCA3/MJD diagnosis was missing, case reports, case series (without controls), reviews, comments, editorials, and guidelines, and studies written in languages other than English were excluded. Neuroimaging studies were addressed in a recent systematic review (Klaes et al., 2016), and therefore were not included in this review.

Clinical rating scales or scores for cerebellar ataxia and studies whose design was intended to identify a trait biomarker - for instance, studies searching for modifiers of age at onset - were not within the scope of this review.

\section{Study organization}

Results were presented in two groups of candidate biomarkers: biological fluid compounds and neurophysio- 
logy characteristics. The main scientific queries were related to evidences on validation against a clinical scale, responsiveness, and clinical significance. If already estimated, sample sizes for future trials were mentioned as well.

\section{Sensitivity to change}

Cohen's Effect Size (CES) or the Standardized Response Mean (SRM) were provided to candidate biomarkers, when available. The following formulas were applied: (1) mean score change/standard deviation (SD) of score at baseline (for CES), and (2) mean score change/SD of score change (for SRM) when data were available and CES, or SRM were not determined.

\section{Results}

\section{Biological fluid compounds}

Table 1 summarizes data on biological fluid compounds reported on SCA3/MJD and included in the present review. Studies with positive results related to disease state, on neurotrophic/growth factors, inflammatory mediators, and astrocyte activators, markers of neuronal and glial loss, oxidative stress, and protein quality control systems markers are described below. Longitudinal data was available only for eotaxin levels, and the effect size of this candidate is described in Figure 1.

Among compounds associated to symptomatic status of SCA3/MJD carriers, only serum neuron-specific enolase
(NSE) levels and glutathione peroxidase activity (GSH-Px) were found to be related to SCA3/MJD by two independent case/control studies each (Tort et al., 2005; Zhou et al., 2011; Pacheco et al., 2013; de Assis et al., 2017). NSE is a peripheral marker of neuronal disruption, and increased levels of this protein are associated to neuronal death. However, inconsistent associations were found between NSE and clinical scales (Table 1). GSH-Px activity reflects antioxidant defense capacity. A moderate inverse correlation of this marker was shown with NESSCA, and differences were observed between symptomatic and presymptomatic phases of the disease (de Assis et al., 2017).

Some biological fluid compounds were associated to SCA3/MJD or to disease severity by single studies using unbiased approaches. Pro-inflammatory factors were particularly prominent among them. After a transcriptomewide gene expression profile approach, quantitative PCR (qPCR) confirmed upregulation of FCGR3B and SELPLG in SCA3/MJD, and the first one was related to disease duration (Raposo et al., 2015). Another unbiased approach analyzed microRNAs (miRs) of peripheral blood samples. miRs are post-transcriptional repressors that can regulate gene expression at different levels. The expression of four specific miRs was found to be up- or down-regulated in SCA3/MJD patients; some of them being involved in astrocyte proliferation. Of note, a down-regulated expression pattern of miR-25 and miR125b was associated to longer disease duration (Shi et al., 2014). Another unbiased approach evaluated serum cytokines levels and higher levels
A

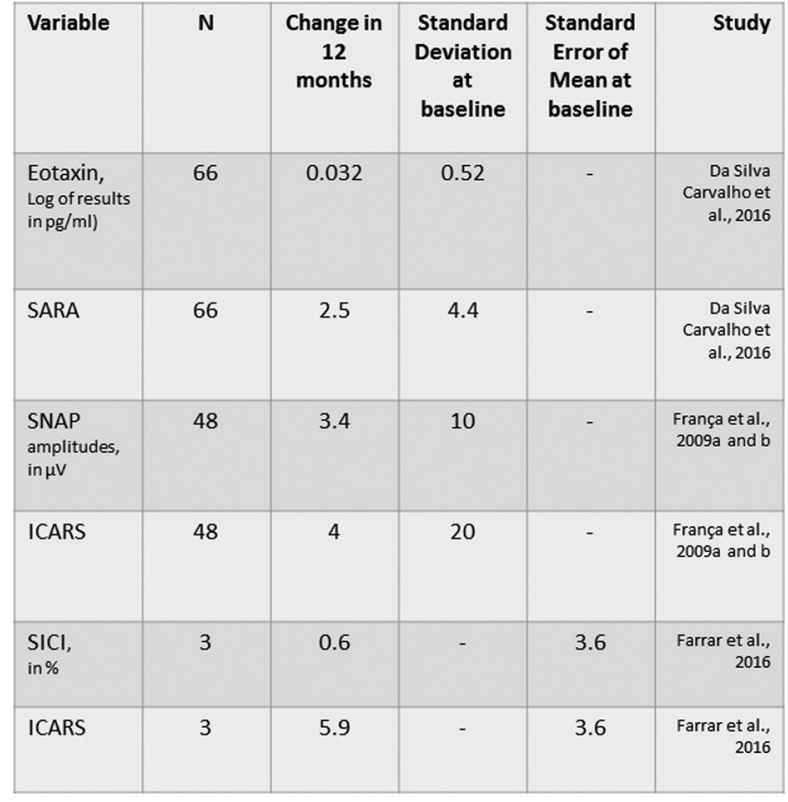

B

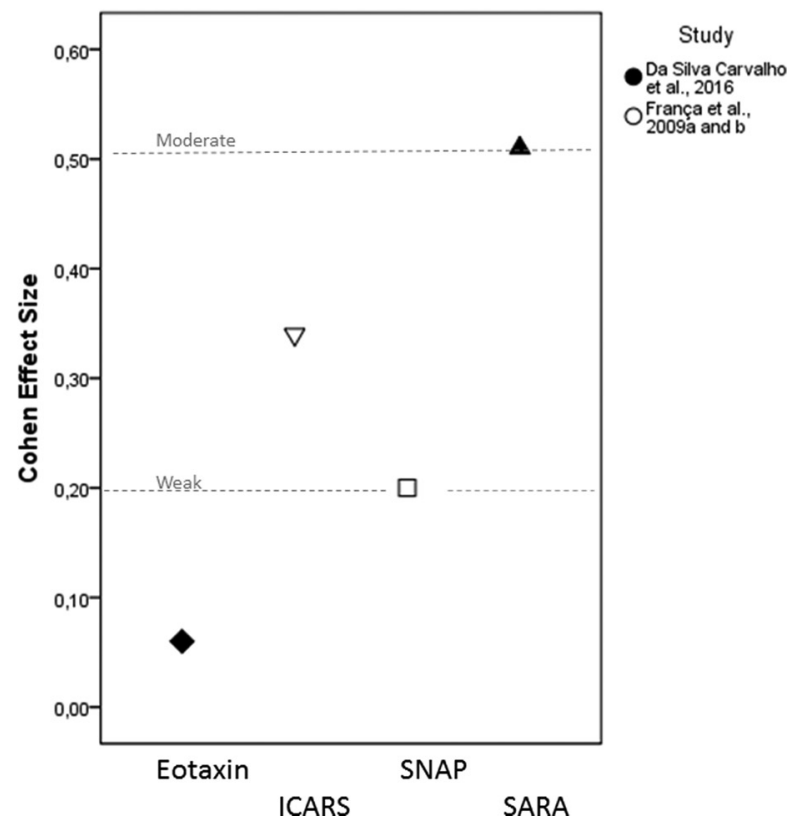

Figure 1 - Candidate biomarkers that have been followed longitudinally in SCA3/MJD subjects. (A) Summary of the longitudinal data obtained for eotaxin and Scale for Assessment and Rating of Ataxia (SARA); sensory nerve action potential (SNAP) amplitudes of sural nerves and International Cooperative Ataxia Rating Scale (ICARS); and short-interval intracortical inhibition (SICI) of motor evoked potentials and ICARS. (B) Cohen effect sizes, when available or when estimation was possible. 


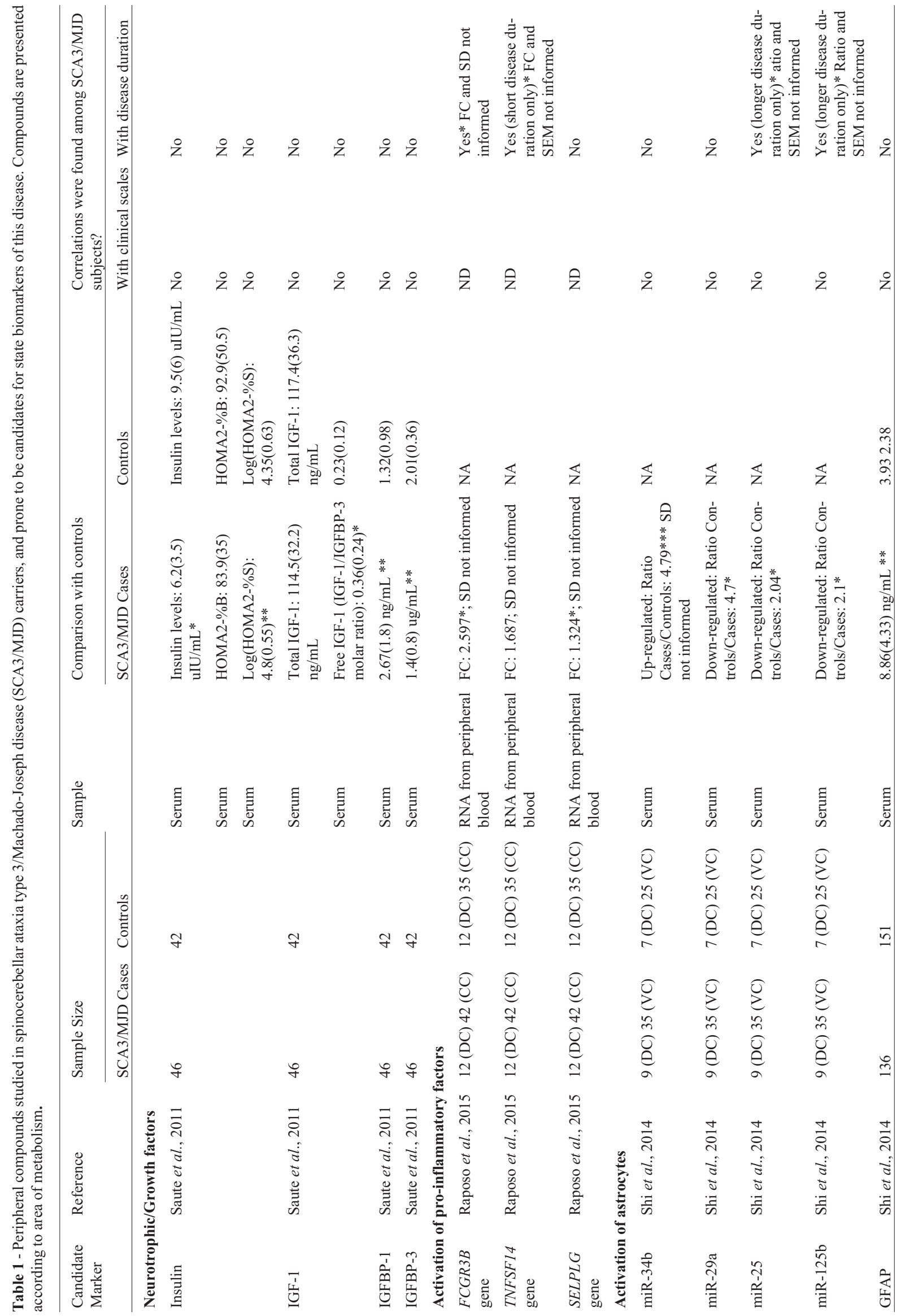




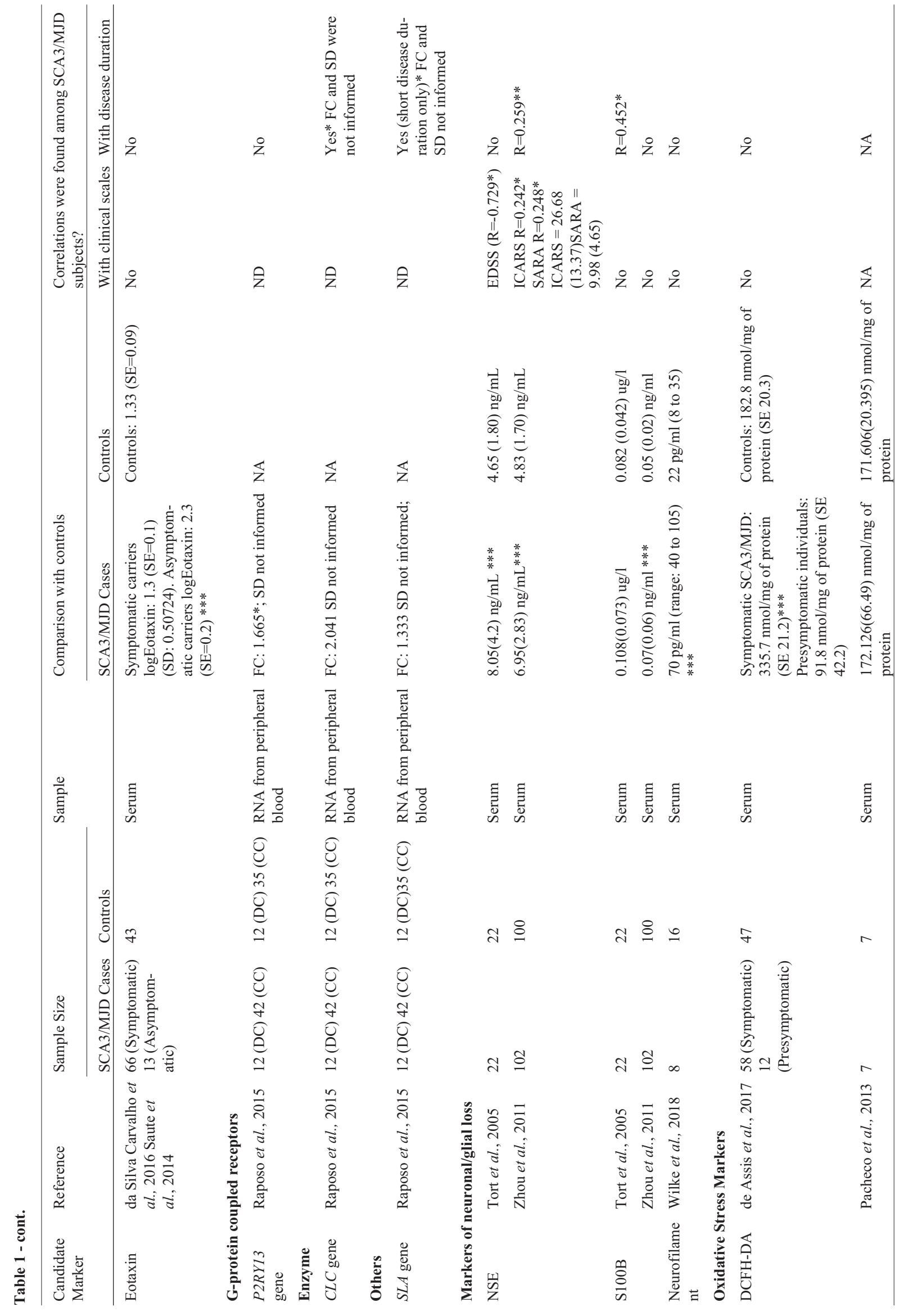




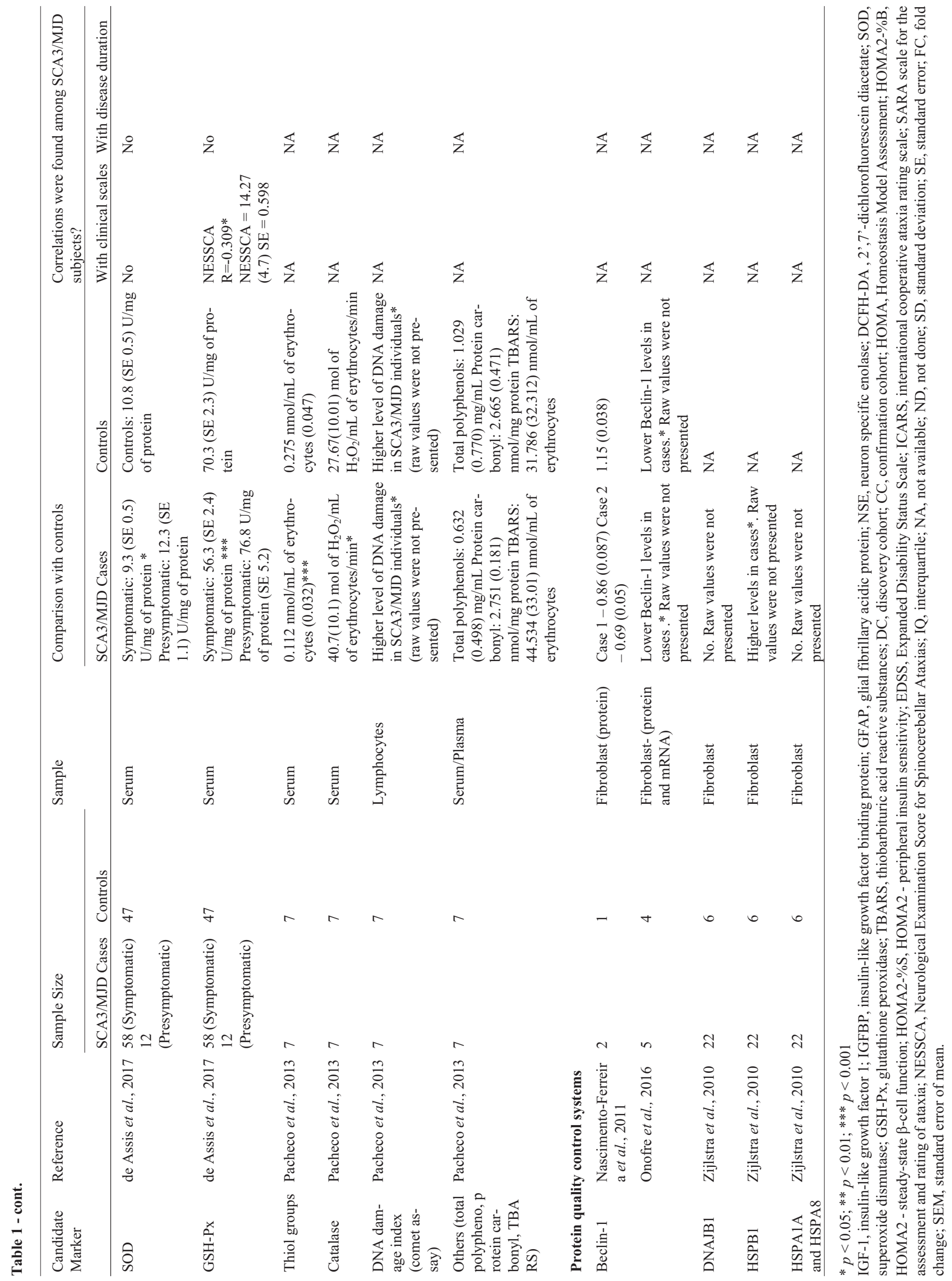




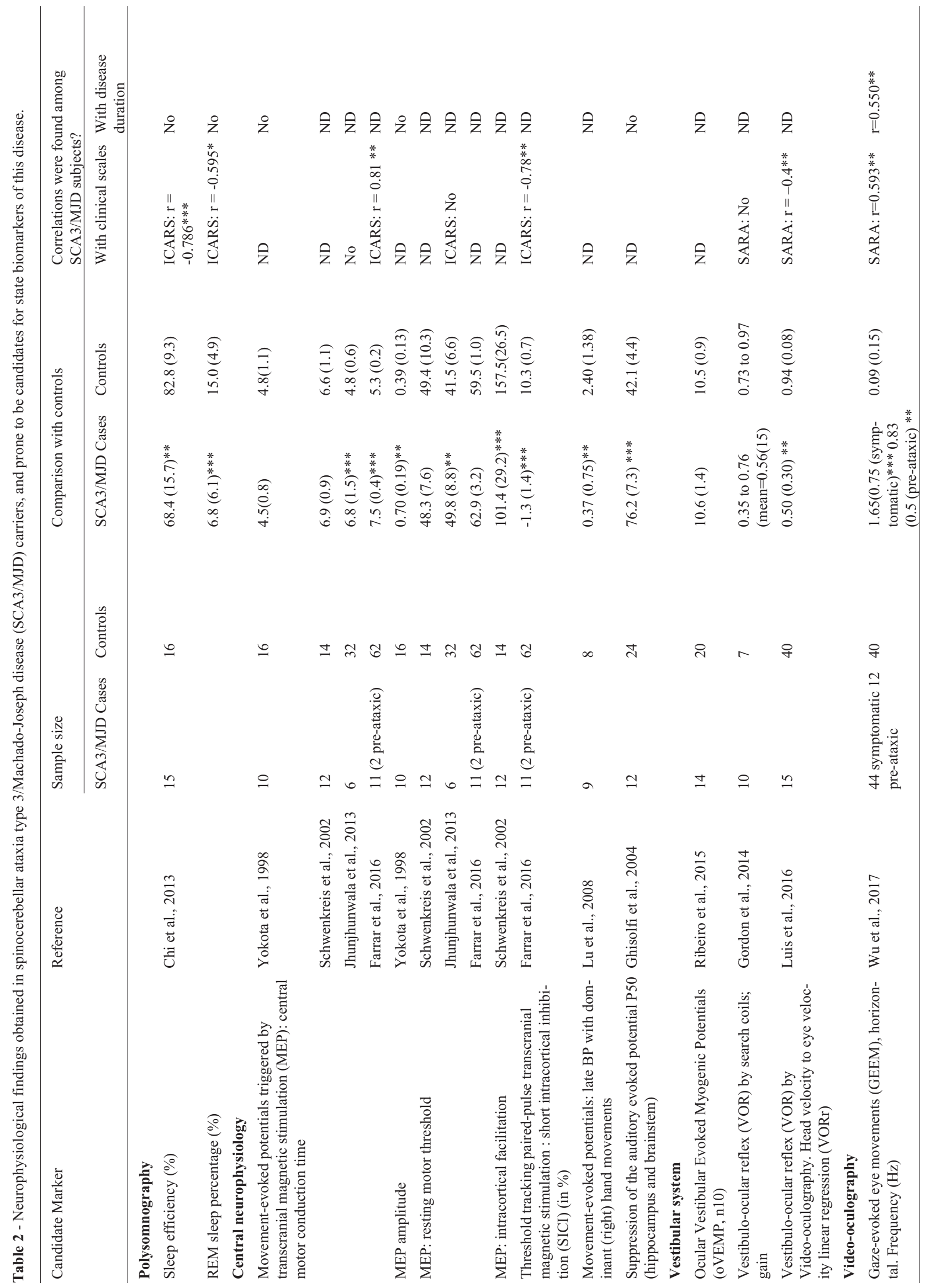




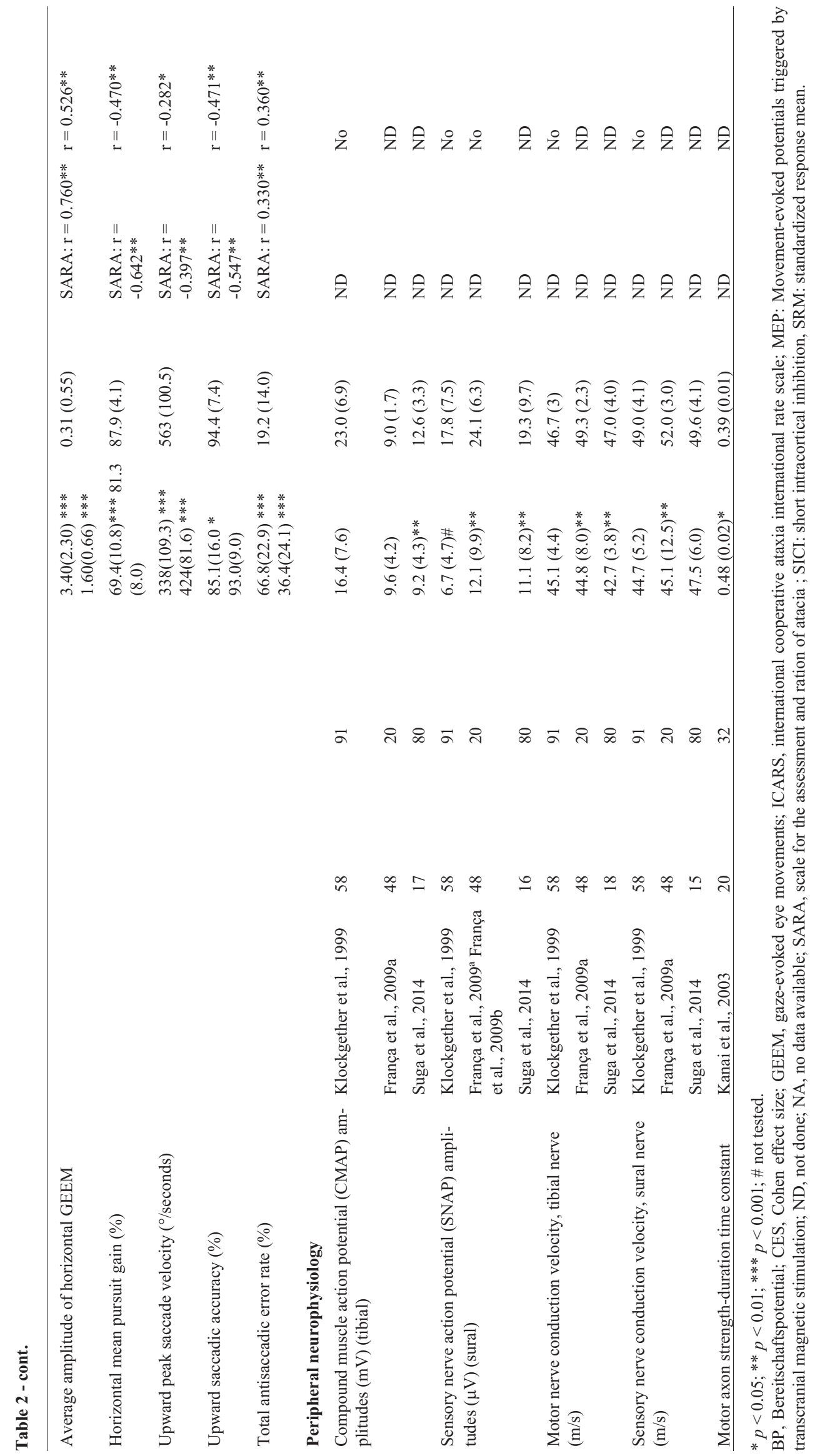


of serum eotaxin, a cytokine secreted by eosinophils and related to astrocytes in central nervous system (CNS). These were found in asymptomatic carriers when compared to both symptomatic patients and controls. A reduction in the levels of this protein was demonstrated in the symptomatic period a year later (da Silva Carvalho et al., 2016). Eotaxin levels and SARA scores obtained simultaneously in these carriers (Saute et al., 2014) were both broadly dispersed, but the ES of Eotaxin (0.06) was smaller than the ES of SARA (0.50) (Figure 1).

\section{Neurophysiology}

Table 2 summarizes data on neurophysiological candidates found by the present literature review. Longitudinal data was available for one parameter of motor evoked potentials (MEP) and for one parameter of peripheral neurophysiology, but the effect size could be estimated for the latter only (Figure 1).

\section{Central neurophysiology}

Motor evoked potentials (MEP) evaluate pyramidal tract conductivity by MEP-derived parameters, such as central motor conduction time (CMCT), amplitude, and resting threshold. CMCT in SCA3/MJD was found to be prolonged and associated to clinical scales by some studies (Jhunjhunwala et al., 2013; Farrar et al., 2016). Cortical activity related to movement preparation and execution, and signs of cortical dysfunction in resting motor threshold, short-interval intracortical inhibition (SICI), and cortical silent period duration were found by a recent study, even in presymptomatic SCA3/MJD individuals (Farrar et al., 2016). These markers were strongly correlated to ICARS. Data on SICI and ICARS progression in 18 months were given in mean and standard error of mean. Therefore, CES could not be estimated (Figure 1).

Among sensory evoked potentials, visual evoked potentials (VEPs), brainstem auditory-evoked response (BAER), somatosensory-EPs (SSEPs), pain-related evoked potentials, and sensory gating at hippocampus/brainstem were already studied in SCA3/MJD, and no good candidate has arisen as a state biomarker (Table 2).

\section{Video-oculography}

Diplopia is a very common finding in patients with SCA3/MJD and can be attributed to ophthalmoplegia or vergence abnormalities. While ophthalmoplegia is easily detected in symptomatic phases of disease, subtle findings such as gaze-evoked and rebound nystagmus, square-wave jerks, saccadic hypermetria, and impaired ocular pursuit are measurable abnormalities described not only in symptomatic (Buttner et al., 1998; Ghasia et al., 2016), but also in presymptomatic carriers (Jacobi et al., 2013; Raposo et al., 2014). Quantitative oculomotor findings have been recently described through video-oculography (Wu et al.,
2017). Several parameters were studied, and most of them were shown to be significantly disturbed even in preclinical phases of disease, and to be related to DD and to SARA in later phases (Table 2). A stepwise worsening from preataxic to symptomatic carriers were seen in the frequency and average amplitude of horizontal gaze-evoked eye movements, upward peak saccade velocity, and total antisaccadic error rates. The lowest dispersion rates in pre-ataxic and symptomatic groups were obtained when measuring the upward peak saccade velocity.

\section{Vestibular system}

Vertigo and imbalance when turning the head are frequent complaints in SCA3/MJD, pointing to involvement of the vestibular system. Measurement of myogenic potentials in the ipsilateral sternocleidomastoid muscle after loud monaural clicks, and of vestibulo-ocular reflex (VOR) after a head impulse test (HIT) were among the neurophysiological evaluations of vestibular dysfunction. VOR disturbances after HIT have been described for a long time in SCA3/MJD (Buttner et al., 1998; Gordon et al., 2003). VOR registrations were improved by using magnetic search coils (Gordon et al., 2014), and video-oculography (VOG) portable systems turned quantitative testing of the VOR possible at the bedside (Agrawal et al., 2014). In a recent study, VOR gain in SCA3/MJD subjects was significantly lower than in controls and correlated with SARA scores in the overall group of ataxic disorders (Luis et al., 2016). VOR dispersion seemed to be larger than SARA dispersion in SCA3/MJD group (Table 2).

\section{Peripheral neurophysiology}

SCA3/MJD has been associated with axonal neuropathy of both motor and sensory nerve fibers, detected by marked reductions of compound muscle (CMAP) and sensory nerve action potential (SNAP) amplitudes. In addition to sensory losses, muscle cramps might be related to this process, being due to the electrical irritability of unmyelinated nerve twigs, enhanced by collateral sprouting secondary to loss of motoneurons. This electrical irritability of unmyelinated nerve twigs was studied once, and further clarification on this disorder is required (Kanai et al., 2003).

Axonal neuropathy in SCA3/MJD is most probably a neuronopathy rather than a distal axonopathy (Kanai et al., 2003; Escorcio Bezerra et al., 2013), and CMAP and SNAP amplitudes are considered indirect measures of the number of peripheral axons. Axonal neuropathy was mainly explained by age in SCA3/MJD (França et al., 2009a; Klockgether et al., 1999; Linnemann et al., 2016). In a longitudinal observation, sural SNAP showed a significant deterioration after 13 months (França et al., 2009a). The CES of SNAP (0.34) was a little higher than CES of ICARS (0.20) obtained in the same period (França et al., 2009b) (Figure 1). 


\section{Discussion}

Several biological fluid compounds and neurophysiological parameters described in SCA3/MJD subjects seemed to be good candidates, but are far from being validated as surrogate state markers for this condition. Most publications described case-control observations where cases were already symptomatic. In contrast, altered results of the peripheral levels of eotaxin and for video-oculography were already found in pre-symptomatic states. Some candidates were associated with disease duration after symptoms onset. The oxidative stress marker GSH-Px, movement-evoked potentials, vestibulo-ocular reflex (VOR), and several video-oculography parameters correlated reasonably and significantly with clinical scales, at this same stage. Only three studies presented a longitudinal design, but no candidate marker was tested in the context of a clinical trial. Validation against a meaningful clinical endpoint was done in some studies. Rate of change in time was obtained for peripheral eotaxin measurements, SICI, and SNAP amplitudes. Although responsiveness to change was not evaluated by the original studies, published parameters permitted us to roughly estimate CES for eotaxin and SNAP. Those values were worse than the ones obtained for the clinical scales (ICARS, SARA and NESSCA) applied simultaneously. It is worth emphasizing that the number of studies that have been designed with the specific aim of identifying biomarkers is extremely limited in this disorder. We could have added other inclusion criteria to our review, such as sample size, existence of technical validation and of a validation cohort, and statistical adjustments in relation to age or gender. Since these additional inclusion criteria would narrow our results, we chose to summarize these and other characteristics in Tables 1 and 2, letting the reader judge about the candidates value for future studies.

SCA3/MJD is a disease essentially confined to the central nervous system. Biological fluid compounds might theoretically reflect the burden of damage related to the disease if they either cross the blood-brain barrier, or are activated both in the CNS and in the periphery. In any case, the search for peripheral compounds is justified by their feasibility in the clinical setting. Although SCA3/MJD pathogenesis is not thoroughly understood and pitfalls might occur in choosing candidates for biomarkers (Aronson, 2005), several clues were already established and are prone to be followed by laboratory studies. Three unbiased surveys aimed to find upregulated genes (Raposo et al., 2015), microRNAs differentially expressed (Shi et al., 2014), and cytokine patterns (da Silva Carvalho et al., 2016) in SCA3/MJD carriers. Preliminary evidence of the first two studies associated overexpression of pro-inflammatory factors FCGR3B and TNFSF14 and the protein encoded by $C L C$ to SCA3/MJD, a pattern that subsides with late phases of disease. Furthermore, down-regulation of microRNAs (miR-25 and miR-125b) was associated with activation of astrocytes that got even worse in late phases of the disease.
Accuracy and reproducibility have not been established to date for mRNA and miRNA expression analyses, and data were presented as fold change or expression ratios. Moreover, potential superiority of effect sizes cannot be inferred, since dispersion measurements (SE, SEM or SD) and relation to clinical scales were not available.

At least three serum measurements showed interesting characteristics: the already mentioned eotaxin, as well as NSE and GSH-Px (Tort et al., 2005; Zhou et al., 2011; da Silva Carvalho et al., 2016; de Assis et al., 2017). Eotaxin is a peptide secreted not only in peripheral tissues by $\mathrm{T}$ lymphocytes, but also by astrocytes in the CNS (da Silva Carvalho et al., 2016). In the unbiased study on cytokines in SCA3/MJD, eotaxin levels were significantly higher in asymptomatic than in symptomatic carriers or in controls. Although neither correlated to clinical scales nor to disease duration at baseline, eotaxin levels were reduced after 360 days in symptomatic carriers. Eotaxin patterns were in line with results of the microRNA study (Shi et al., 2014), and both unbiased studies raised the hypothesis of astrocyte activation in SCA3/MJD, possibly present in pre-clinical phases, and evolving to exhaustion as the disease progresses. Although eotaxin effect size was small in symptomatic carriers (Figure 1), the effect size in preclinical phases remains unknown. The peripheral indicator of ongoing neuronal damage NSE has been evaluated by two different studies on SCA3/MJD (Tort et al., 2005; Zhou et al., 2011). Increased serum levels of NSE were described by both publications, and the larger study was able to associate NSE to disease duration. In contrast, NSE levels were inversely related to the Extended Disability Status Scale of Kurtzke (EDSS) in the older, and directly related to ICARS and SARA in the more recent study. While this discrepancy remains unsolved, the application of NSE as a potential biomarker is precluded. The activity of the antioxidant enzyme glutathione peroxidase (GSH-Px) was low in SCA3/MJD symptomatic individuals in two studies (Pacheco et al., 2013; de Assis et al., 2017). GSH-Px differences from symptomatic to presymptomatic phases of the disease suggested a temporal association of lower GSH-Px activity to more advanced disease stages, sustaining some expectation in this candidate marker.

Neurophysiological studies have been done based on the hypothesis that the underlying neurological function under study is relevant for SCA3/MJD symptomatology. However, important findings associated to this disease are related to cerebellum and cerebellar-brainstem connections. There is no bedside tool to measure electrophysiological manifestations of cerebellar dysfunction. In spite of that, promising markers emerged from neurophysiology. Among the parameters obtained from MEP, central motor conduction time and SICI were significantly changed and related to ICARS in symptomatic carriers (Figure 1). SICI variability was very large, suggesting that potential CES would be small, for future trials addressed to pyramidal in- 
volvement in this disease. VOR is affected in SCA3/MJD symptomatic carriers, and showed a moderate association to SARA, with similar measures of dispersion. Peripheral nerve studies have been performed as well, and sural SNAP showed a significant deterioration after 13 months (França et al., 2009a,b). We were able to estimate CES of both SNAP and ICARS, 0.34 and 0.20, respectively (Figure 1). SARA CES (0.50) was superior to both.

Since they portray brainstem dysfunction, neurophysiological measurements of eye movement abnormalities are very interesting candidate biomarkers. A promising case-control study reported that frequency and amplitude of gaze evoked nystagmus, smooth pursuit eye movements (gain), upward peak velocity and accuracy of saccades, and error rates of antisaccades were already affected in preclinical phases of the disease, and were all related to SARA scores and to disease duration in symptomatic carriers $(\mathrm{Wu}$ et al., 2016). This results scenario suggests that these manifestations decline in SCA3/MJD in a progressive manner. Although SD of SARA scores was not presented, other observations described SD as being equivalent to $40 \%$ to $60 \%$ of SARA average results (Jacobi et al., 2011, 2015; Ashizawa et al., 2013; Saute et al., 2014). Some video-oculographic parameters obtained in SCA3/MJD subjects showed proportionally smaller SDs than these figures, like horizontal mean pursuit gain and upward saccadic accuracy (Table 2).

Although evidence levels remain preliminary, the paragraphs below address promising additional biomarkers due to their direct roles in the SCA3/MJD pathophysiology. Molecules associated to quality control systems might play a very relevant role in SCA3/MJD, and we can highlight here two promising ones: beclin-1 and DNAJB1. Beclin-1 is a marker of protein quality control systems, and low protein as well as mRNA levels were found in fibroblasts from symptomatic SCA3/MJD individuals (Nascimento-Ferreira et al., 2011; Onofre et al., 2016). DNAJB1 is a molecular chaperone that stimulates the ATPase activity of Hsp70 heat-shock proteins in order to promote protein folding and prevent misfolded protein aggregation. High DNAJB1 levels were associated with earlier ages at onset than those predicted by the CAG repeat length (Zijlstra et al., 2010). Both compounds should be further evaluated using larger sample sizes and by performing longitudinal observations.

Soluble mutant ataxin-3 levels were measured by time-resolved Forster resonance energy transfer (TRFRET) immunoassay in human cell lines and brain samples of transgenic SCA3/MJD mice model (Nguyen et al., 2013), but properties of soluble ataxin-3 as a disease biomarker were not addressed up to date. Soluble mutant protein levels have been measured in other neurodegenerative disorders, such as in Huntington disease (HD), and were associated to clinical features (Moscovitch-Lopatin et al., 2013). Soluble huntingtin is currently being evaluated as an outcome in recent HD clinical trials (Huntington Study Group Reach2HD Investigators, 2015; Süssmuth et al., 2015). Likewise measurements of soluble mutant ataxin-3 should be evaluated in future longitudinal studies on SCA3/MJD.

Finally, it is worth to stress that biomarkers are mostly needed for the pre-clinical phases of SCA3/MJD. The pathological process is already on the way before the onset of gait ataxia, and future therapies will probably be more effective if starting early. Studies on pre-symptomatic carriers face more difficulties than others, such as lack of adherence and ethical issues. Fortunately, the time burden measured by the concept "disease duration" since the onset of symptoms and useful for symptomatic studies, can be solved by equations that predict the age at onset and that have recently appeared in the literature (Tezenas du Montcel et al., 2014; Mattos et al., 2019). They will help validating biomarkers for the pre-symptomatic phases.

In conclusion, several potential candidates as state biomarkers have been preliminarily described, albeit through a majority of studies without good sample sizes and/or rigorous designs for the validation of such biomarkers. Candidates for surrogate biomarkers of the presymptomatic state were even more scarcely described in the literature. Studies on pre-clinical phases, such as those performed on cytokines and on neurophysiological measurements of eye movement abnormalities, are even more important, since most clinical scales give normal scores in this period. Prospective evaluations are required for all of them, together with measurements of clinical scales and of PGIs. Validation against a MCID, rate of change in time, and responsiveness to change should be established. We are aware that several barriers can delay this goal, including restraints that go beyond the scientists' efforts and patients' goodwill. For example, neurophysiology, molecular, and neuroimaging data depend upon technology companies, where planned obsolescence is intrinsic to the production lines. The constant change in platforms turns all knowledge acquisition longer and harder than expected. Hence, solutions for these dilemmas have to be searched for and the future needs to be carefully planned. To this, all-embracing, multi-center studies can be the answer.

\section{Acknowledgments}

$\mathrm{CMO}$ and GB were supported by Coordenação de Aperfeiçoamento de Pessoal de Nível Superior - Brasil (CAPES) - Finance Code 001. GVF, MLSP and LBJ were supported by Conselho Nacional de Desenvolvimento Científico e Tecnológico (CNPq).

\section{Conflict of interest}

The authors declare no conflict of interest that could be perceived as prejudicial to the impartiality of the reported research. 


\section{Author Contributions}

LBJ conceived the study; CMO and LBJ contributed to section "Results: Neurophysiology"; GVF, GB, JAMS, MLSP and LBJ contributed to section "Results: Biological fluid compounds"; all authors contributed to sections "Material and Methods" and "Discussion"; all authors read and approved the submitted version of the manuscript.

\section{References}

Agrawal Y, Schubert MC, Migliaccio AA, Zee DS, Schneider E, Lehnen N and Carey JP (2014) Evaluation of quantitative head impulse testing using search coils versus video-oculography in older individuals. Otol Neurotol 35:283-288.

Aronson JK (2005) Biomarkers and surrogate endpoints. Br J Clin Pharmacol 59:491-494.

Ashizawa T, Figueroa KP, Perlman SL, Gomez CM, Wilmot GR, Schmahmann JD, Ying SH, Zesiewicz TA, Paulson HL, Shakkottai VG et al. (2013) Clinical characteristics of patients with spinocerebellar ataxias 1, 2, 3 and 6 in the US; a prospective observational study. Orphanet J Rare Dis 8:177.

Buttner N, Geschwind D, Jen JC, Perlman S, Pulst SM and Baloh RW (1998) Oculomotor phenotypes in autosomal dominant ataxias. Arch Neurol 55:1353-1357.

Chan E, Charles P, Ribai P, Goizet C, Marelli C, Vincitorio CM, Le Bayon A, Guyant-Maréchal L, Vandenberghe N, Anheim M et al. (2011) Quantitative assessment of the evolution of cerebellar signs in spinocerebellar ataxias. Mov Disord 26:534-538.

Chi NF, Shiao GM, Ku HL and Soong BW (2013) Sleep disruption in spinocerebellar ataxia type 3: a genetic and polysomnographic study. J Chin Med Assoc. 76:25-30.

da Silva Carvalho G, Saute JA, Haas CB, Torrez VR, Brochier AW, Souza GN, Furtado GV, Gheno T, Russo A, Monte TL et al. (2016) Cytokines in Machado Joseph Disease/Spinocerebellar Ataxia 3. Cerebellum 15:518-525.

de Assis AM, Saute JAM, Longoni A, Haas CB, Torrez VR, Brochier AW, Souza GN, Furtado GV, Gheno TC, Russo A et al. (2017) Peripheral oxidative stress biomarkers in spinocerebellar ataxia type 3/Machado-Joseph disease. Front Neurol 8:485.

de Castilhos RM, Furtado GV, Gheno TC, Schaeffer P, Russo A, Barsottini O, Pedroso JL, Salarini DZ, Vargas FR, de Lima MA et al. (2014) Spinocerebellar ataxias in Brazil - frequencies and modulating effects of related genes. Cerebellum 13:17-28.

du Montcel ST, Charles P, Ribai P, Goizet C, Le Bayon A, Labauge P, Guyant-Maréchal L, Forlani S, Jauffret C, Vandenberghe $\mathrm{N}$ et al. (2008) Composite cerebellar functional severity score: Validation of a quantitative score of cerebellar impairment. Brain 131:1352-1361.

du Montcel ST, Durr A, Bauer P, Figueroa KP, Ichikawa Y, Brussino A, Forlani S, Rakowicz M, Schöls L, Mariotti C et al. (2014) Modulation of the age at onset in spinocerebellar ataxia by CAG tracts in various genes. Brain 137:24442455.

Duarte-Silva S and Maciel P (2018) Pharmacological therapies for Machado-Joseph disease. Adv Exp Med Biol 1049:369394.
Dürr A, Stevanin G, Cancel G, Duyckaerts C, Abbas N, Didierjean O, Chneiweiss H, Benomar A, Lyon-Caen O, Julien J et al. (1996) Spinocerebellar ataxia 3 and Machado-Joseph disease: Clinical, molecular, and neuropathological features. Ann Neurol 39:490-499.

Escorcio Bezerra ML, Pedroso JL, Pinheiro DS, Braga-Neto P, Povoas Barsottini OG, Braga NI and Manzano GM (2013) Pattern of peripheral nerve involvement in Machado-Joseph disease: neuronopathy or distal axonopathy? A clinical and neurophysiological evaluation. Eur Neurol 69:129-133.

Farrar MA, Vucic S, Nicholson G and Kiernan MC (2016) Motor cortical dysfunction develops in spinocerebellar ataxia type 3. Clin Neurophysiol 127:3418-3424.

França Jr MC, D'abreu A, Nucci A, Cendes F and Lopes-Cendes I (2009a) Prospective study of peripheral neuropathy in Machado-Joseph disease. Muscle Nerve 40:1012-1018.

França Jr MC, D'Abreu A, Nucci A, Cendes F and Lopes-Cendes I (2009b) Progression of ataxia in patients with MachadoJoseph disease. Mov Disord 24:1387-1390.

Ghasia FF, Wilmot G, Ahmed A and Shaikh AG (2016) Strabismus and micro-opsoclonus in Machado-Joseph disease. Cerebellum 15:491-497.

Ghisolfi ES, Maegawa GH, Becker J, Zanardo AP, Strimitzer IM Jr, Prokopiuk AS, Pereira ML, Carvalho T, Jardim LB and Lara DR (2004) Impaired P50 sensory gating in MachadoJoseph disease. Clin Neurophysiol 115:2231-2235.

Globas C, du Montcel ST, Baliko L, Boesch S, Depondt C, DiDonato S, Durr A, Filla A, Klockgether T, Mariotti C et al. (2008) Early symptoms in spinocerebellar ataxia type 1, 2, 3, and 6. Mov Disord 23:2232-2238.

Gordon CR, Joffe V, Vainstein G and Gadoth N (2003) Vestibulo-ocular areflexia in families with spinocerebellar ataxia type 3 (Machado-Joseph disease). J Neurol Neurosurg Psychiatry $74: 1403-1406$.

Gordon CR, Zivotofsky AZ and Caspi A (2014) Impaired vestibulo-ocular reflex (VOR) in spinocerebellar ataxia type 3 (SCA3): Bedside and search coil evaluation. J Vestib Res 24:351-355.

Huntington Study Group Reach2HD Investigators (2015) Safety, tolerability, and efficacy of PBT2 in Huntington's disease: A phase 2, randomised, double-blind placebo-controlled trial. Lancet Neurol 14:39-47.

Jacobi H, Bauer P, Giunti P, Labrum R, Sweeney MG, Charles P, Dürr A, Marelli C, Globas C, Linnemann C et al. (2011) The natural history of spinocerebellar ataxia type 1,2,3, and 6: A 2-year follow-up study. Neurology 77:1035-1041.

Jacobi H, Reetz K, du Montcel ST, Bauer P, Mariotti C, Nanetti L, Rakowicz M, Sulek A, Durr A, Charles P et al. (2013) Biological and clinical characteristics of individuals at risk for spinocerebellar ataxia types 1, 2, 3, and 6 in the longitudinal RISCA study: Analysis of baseline data. Lancet Neurol 12:650-658.

Jacobi H, du Montcel ST, Bauer P, Giunti P, Cook A, Labrum R, Parkinson MH, Durr A, Brice A, Charles P et al. (2015) Long-term disease progression in spinocerebellar ataxia types 1, 2, 3, and 6: a longitudinal cohort study. Lancet Neurol 14:1101-1108.

Jardim LB, Pereira ML, Silveira I, Ferro A, Sequeiros J and Giugliani R (2001) Neurologic findings in Machado-Joseph disease: Relation with disease duration, subtypes, and (CAG)n. Arch Neurol 58:899-904. 
Jhunjhunwala K, Prashanth DK, Netravathi M, Jain S, Purushottam M and Pal PK (2013) Alterations in cortical excitability and central motor conduction time in spinocerebellar ataxias 1, 2 and 3: A comparative study. Parkinsonism Relat Disord 19:306-311.

Kanai K, Kuwabara S, Arai K, Sung JY, Ogawara K and Hattori T (2003) Muscle cramp in Machado-Joseph disease: Altered motor axonal excitability properties and mexiletine treatment. Brain 126:965-973.

Kieling C, Prestes PR, Saraiva-Pereira ML and Jardim LB (2007) Survival estimates for patients with Machado-Joseph disease (SCA3). Clin Genet 72:543-545.

Kieling C, Rieder CR, Silva AC, Saute JA, Cecchin CR, Monte TL and Jardim LB (2008) A neurological examination score for the assessment of spinocerebellar ataxia 3 (SCA3). Eur J Neurol 15:371-376.

Klaes A, Reckziegel E, Franca MC Jr, Rezende TJ, Vedolin LM, Jardim LB and Saute JA (2016) MR imaging in spinocerebellar ataxias: A systematic review. AJNR Am J Neuroradiol 37:1405-1412.

Klockgether T, Schöls L, Abele M, Bürk K, Topka H, Andres F, Amoiridis G, Lüdtke R, Riess O, Laccone F and Dichgans J (1999) Age related axonal neuropathy in spinocerebellar ataxia type 3/Machado-Joseph disease(SCA3/MJD). J Neurol Neurosurg Psychiatry 66:222-224.

Li X, Liu H, Fischhaber PL and Tang TS (2015) Toward therapeutic targets for SCA3: Insight into the role of Machado-Joseph disease protein ataxin-3 in misfolded proteins clearance. Prog Neurobiol 132:34-58.

Linnemann C, Tezenas du Montcel S, Rakowicz M, SchmitzHübsch T, Szymanski S, Berciano J, van de Warrenburg BP, Pedersen K, Depondt C, Rola R et al. (2016) Peripheral neuropathy in spinocerebellar ataxia type 1,2,3, and 6. Cerebellum 15:165-173.

Lu MK, Shih HT, Huang KJ, Ziemann U, Tsai CH, Chang FC, Chen YC, Lin YT, Huang WS, Lee CC et al. (2008) Movement-related cortical potentials in patients with MachadoJoseph disease. Clin Neurophysiol 119:1010-1019.

Luis L, Costa J, Muñoz E, de Carvalho M, Carmona S, Schneider E, Gordon CR and Valls-Solé J (2016) Vestibulo-ocular reflex dynamics with head-impulses discriminates spinocerebellar ataxias types 1, 2 and 3 and Friedreich ataxia. J Vestib Res 26:327-334.

Matos CA, Carmona V, Vijayakumar UG, Lopes S, Albuquerque $\mathrm{P}$, Conceição $\mathrm{M}$, Nobre RJ, Nóbrega $\mathrm{C}$ and de Almeida LP (2018) Gene therapies for polyglutamine diseases. Adv Exp Med Biol 1049:395-438.

Mattos EP, Leotti VB, Soong BW, Raposo M, Lima M, Vasconcelos J, Fussiger H, Souza GN, Kersting N, Furtado GV et al. (2019) Age at onset prediction in spinocerebellar ataxia type 3 changes according to population of origin. Eur Jour Neurol 26:113-120.

Moscovitch-Lopatin M, Goodman RE, Eberly S, Ritch JJ, Rosas HD, Matson S, Matson W, Oakes D, Young AB, Shoulson I et al. (2013) HTRF analysis of soluble huntingtin in PHAROS PBMCs. Neurology 81:1134-1140.

Nascimento-Ferreira I, Santos-Ferreira T, Sousa-Ferreira L, Auregan G, Onofre I, Alves S, Dufour N, Colomer Gould VF, Koeppen A, Déglon N et al. (2011) Overexpression of the autophagic beclin-1 protein clears mutant ataxin-3 and alleviates Machado-Joseph disease. Brain 134:1400-1415.
Nguyen HP, Hübener J, Weber JJ, Grueninger S, Riess O and Weiss A (2013) Cerebellar soluble mutant ataxin-3 level decreases during disease progression in Spinocerebellar Ataxia Type 3 mice. PLoS One 8:e62043.

Onofre I, Mendonça N, Lopes S, Nobre R, de Melo JB, Carreira IM, Januário C, Gonçalves AF and de Almeida LP (2016) Fibroblasts of Machado Joseph Disease patients reveal autophagy impairment. Sci Rep 6:28220.

Pacheco LS, da Silveira AF, Trott A, Houenou LJ, Algarve TD, Belló C, Lenz AF, Mânica-Cattani MF and da Cruz IB (2013) Association between Machado-Joseph disease and oxidative stress biomarkers. Mutat Res 757:99-103.

Raposo M, Vasconcelos J, Bettencourt C, Kay T, Coutinho P and Lima M (2014) Nystagmus as an early ocular alteration in Machado-Joseph disease (MJD/SCA3). BMC Neurol 14:17.

Raposo M, Bettencourt C, Maciel P, Gao F, Ramos A, Kazachkova N, Vasconcelos J, Kay T, Rodrigues AJ, Bettencourt B et al. (2015) Novel candidate blood-based transcriptional biomarkers of Machado-Joseph disease. Mov Disord 30:968-975.

Ribeiro RS, Pereira MM, Pedroso JL, Braga-Neto P, Barsottini OG and Manzano GM (2015) Cervical and ocular vestibular evoked potentials in Machado-Joseph disease: Functional involvement of otolith pathways. J Neurol Sci 358:294-298.

Saute JA, da Silva AC, Muller AP, Hansel G, de Mello AS, Maeda F, Vedolin L, Saraiva-Pereira ML, Souza DO, Arpa J et al. (2011) Serum insulin-like system alterations in patients with spinocerebellar ataxia type 3. Mov Disord 26:731-735.

Saute JA, Donis KC, Serrano-Munuera C, Genis D, Ramirez LT, Mazzetti P, Pérez LV, Latorre P, Sequeiros J, Matilla-Dueñas A et al. (2012) Ataxia rating scales - psychometric profiles, natural history and their application in clinical trials. Cerebellum 11:488-504.

Saute JA, de Castilhos RM, Monte TL, Schumacher-Schuh AF, Donis KC, D’Ávila R, Souza GN, Russo AD, Furtado GV, Gheno TC et al. (2014) A randomized, phase 2 clinical trial of lithium carbonate in Machado-Joseph disease. Mov Disord 29:568-573.

Saute JA, Rieder CR, Castilhos RM, Monte TL, SchumacherSchuh AF, Donis KC, D’Ávila R, Souza GN, Russo AD, Furtado GV et al. (2015) Planning future clinical trials in Machado Joseph disease: Lessons from a phase 2 trial. J Neurol Sci 358:72-76.

Saute JA and Jardim LB (2015) Machado Joseph disease: clinical and genetic aspects, and current treatment. Expert Opinion on Orphan Drugs 3:517-535.

Schmitz-Hübsch T, du Montcel ST, Baliko L, Berciano J, Boesch S, Depondt C, Giunti P, Globas C, Infante J, Kang JS et al. (2006) Scale for the assessment and rating of ataxia: Development of a new clinical scale. Neurology 66:1717-1720.

Schmitz-Hübsch T, Coudert M, Bauer P, Giunti P, Globas C, Baliko L, Filla A, Mariotti C, Rakowicz M, Charles P et al. (2008) Spinocerebellar ataxia types 1, 2, 3, and 6: Disease severity and nonataxia symptoms. Neurology 71:982-989.

Schmitz-Hübsch T, Fimmers R, Rakowicz M, Rola R, Zdzienicka E, Fancellu R, Mariotti C, Linnemann C, Schöls L, Timmann $\mathrm{D}$ et al. (2010) Responsiveness of different rating instruments in spinocerebellar ataxia patients. Neurology 74:678-684.

Schöls L, Amoiridis G, Büttner T, Przuntek H, Epplen JT and Riess O (1997) Autosomal dominant cerebellar ataxia: phe- 
notypic differences in genetically defined subtypes? Ann Neurol 42:924-932.

Schwenkreis P, Tegenthoff M, Witscher K, Börnke C, Przuntek H, Malin JP and Schöls L (2002) Motor cortex activation by transcranial magnetic stimulation in ataxia patients depends on the genetic defect. Brain 125:301-309.

Shi Y, Huang F, Tang B, Li J, Wang J, Shen L, Xia K and Jiang H (2014) MicroRNA profiling in the serums of SCA3/MJD patients. Int J Neurosci 124:97-101.

Streiner DL and Norman GR (2008) Health Measurement Scales-A Practical Guide to Their Development and Use. 4th edition. Oxford University Press, Oxford, $431 \mathrm{pp}$.

Suga N, Katsuno M, Koike H, Banno H, Suzuki K, Hashizume A, Mano T, Iijima M, Kawagashira Y, Hirayama $\mathrm{M}$ et al. (2014) Schwann cell involvement in the peripheral neuropathy of spinocerebellar ataxia type 3. Neuropathol Appl Neurobiol 40:628-639.

Süssmuth SD, Haider S, Landwehrmeyer GB, Farmer R, Frost C, Tripepi G, Andersen CA, Di Bacco M, Lamanna C, Diodato E et al. (2015) An exploratory double-blind, randomized clinical trial with selisistat, a SirT1 inhibitor, in patients with Huntington's disease. Br J Clin Pharmacol 79:465-476.

Tang B, Liu C, Shen L, Dai H, Pan Q, Jing L, Ouyang S and Xia J (2000) Frequency of SCA1, SCA2, SCA3/MJD, SCA6, SCA7, and DRPLA CAG trinucleotide repeat expansion in patients with hereditary spinocerebellar ataxia from Chinese kindreds. Arch Neurol 57:540-544.

Tezenas du Montcel S, Durr A, Bauer P, Figueroa KP, Ichikawa Y, Brussino A, Forlani S, Rakowicz M, Schöls L, Mariotti C et al. (2014) Modulation of the age at onset in spinocerebellar ataxia by CAG tracts in various genes. Brain 137:2444-2455.

Tort AB, Portela LV, Rockenbach IC, Monte TL, Pereira ML, Souza DO, Rieder CR and Jardim LB (2005) S100B and NSE serum concentrations in Machado Joseph disease. Clin Chim Acta 351:143-148.

Trouillas P, Takayanagi T, Hallett M, Currier RD, Subramony SH, Wessel K, Bryer A, Diener HC, Massaquoi S, Gomez CM et al. (1997) International Cooperative Ataxia Rating Scale for pharmacological assessment of the cerebellar syn- drome. The Ataxia Neuropharmacology Committee of the World Federation of Neurology. J Neurol Sci 145:205-211.

Wilke C, Bender F, Hayer SN, Brockmann K, Schöls L, Kuhle J and Synofzik M (2018) Serum neurofilament light is increased in multiple system atrophy of cerebellar type and in repeat-expansion spinocerebellar ataxias: a pilot study. J Neurol 265:1618-1624.

Wu C, Chen DB, Feng L, Zhou XX, Zhang JW, You HJ, Liang XL, Pei Z and Li XH (2017) Oculomotor deficits in spinocerebellar ataxia type 3: Potential biomarkers of preclinical detection and disease progression. CNS Neurosci Ther 23:321-328.

Yokota T, Sasaki H, Iwabuchi K, Shiojiri T, Yoshino A, Otagiri A, Inaba A and Yuasa T (1998) Electrophysiological features of central motor conduction in spinocerebellar atrophy type 1, type 2, and Machado-Joseph disease. J Neurol Neurosurg Psychiatry 65:530-534.

Zhou J, Lei L, Shi Y, Wang J, Jiang H, Shen L and Tang B (2011) Serum concentrations of NSE and S100B in spinocerebellar ataxia type 3/Machado-Joseph disease. Zhong Nan Da Xue Xue Bao Yi Xue Ban 36:504-510.

Zhou Q, Ni W, Dong Y, Wang N, Gan SR and Wu ZY (2014) The role of apolipoprotein $\mathrm{E}$ as a risk factor for an earlier age at onset for Machado-Joseph disease is doubtful. PLoS One 9:e111356.

Zijlstra MP, Rujano MA, Van Waarde MA, Vis E, Brunt ER and Kampinga HH (2010) Levels of DNAJB family members (HSP40) correlate with disease onset in patients with spinocerebellar ataxia type 3. Eur J Neurosci 32:760-770.

\section{Internet resources}

WHO International Programme on Chemical Safety (2001) Biomarkers in Risk Assessment: Validity and Validation, http://www.inchem.org/documents/ehc/ehc/ehc222.htm (accessed 17 January 2018)

Associate Editor: Roberto Giugliani

License information: This is an open-access article distributed under the terms of the Creative Commons Attribution License (type CC-BY), which permits unrestricted use, distribution and reproduction in any medium, provided the original article is properly cited. 